\title{
Managing Hypothyroidism in Pediatric Patients
}

\author{
An expert interview with Stephen $\mathrm{H}$ LaFranchi
}

Oregon Health \& Science University, Portland, OR, US

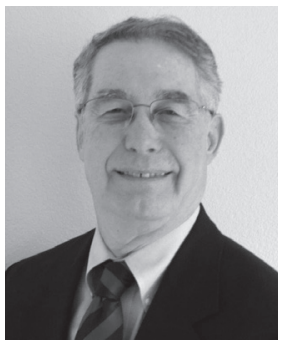

\section{Stephen $\mathbf{H}$ LaFranchi}

Stephen H LaFranchi, MD, is Professor of Pediatrics at the Oregon Health \& Science University. Dr LaFranchi received his MD, Pediatric Residency, and Pediatric Endocrine Fellowship training at University of California, Los Angeles. He joined the faculty at the Oregon Health \& Science University and was Chief of Pediatric Endocrinology and Director of Fellowship Training from 1975-2001. Dr LaFranchi served the Pediatric Endocrine Society as Chair of the Education Committee (1984-1987), treasurer (1988-1993) and then president (1999-2000). He served on the endocrine subboard of the American Board of Pediatrics for 6 years (1993-1998). Dr LaFranchi has been the endocrine consultant to the 6-state Northwest Regional Newborn Screening Program since 1975. He was the coordinating author of the 1993 American Academy of Pediatrics guidelines for management of congenital hypothyroidism. He is the author of 12 pediatric thyroid topics for several online resources and past associate editor of Thyroid.

\section{Keywords}

Congenital hypothyroidism, newborn screening programs, delayed thyroid-stimulating hormone elevation, congenital central hypothyroidism, subclinical hypothyroidism, thyroid hormone treatment, children

Disclosure: Dr LaFranchi has nothing to declare in relation to this article.

Review Process: This is an expert interview and as such has not undergone the journal's usual peer review process but has been approved by the editorial board.

Authorship: The named author meets the Internationa Committee of Medical Journal Editors (ICMJE) criteria for authorship of this manuscript, takes responsibility for the integrity of the work as a whole, and has given final approval to the version to be published.

open Access: This article is published under the Creative Commons Attribution Noncommercial License, which permits any non-commercial use, distribution, adaptation, and reproduction provided the original author(s) and source are given appropriate credit.

Received: July 19, 2018

Published Online: September 12, 2018

Citation: US Endocrinology, 2018;14(2):51-3.

Corresponding Author: Stephen H LaFranchi, Department of Pediatrics [CDRCP], Oregon

Health \& Science University, 707 SW Gaines St,

Portland, Oregon, US. E: lafrancs@ohsu.edu
$\mathrm{T}$ his interview explores current controversies in the management of hypothyroidism in children. Newborn screening programs have allowed early detection and treatment of babies with congenital hypothyroidism, but some programs do not detect cases with delayed thyroidstimulating hormone (TSH) elevation and most do not detect congenital central hypothyroidism. Lack of harmonization of TSH cut-offs means that some programs do not detect milder cases of congenital hypothyroidism. Detection of such mild cases is controversial as most patients have transient hypothyroidism and the benefit of treatment is not proven. The most common disorder of thyroid function in children is subclinical hypothyroidism-many of these cases represent TSH assay artifacts or are associated with obesity, but some will progress to overt hypothyroidism. Some patients/parents are not happy with levothyroxine treatment alone, which raises the consideration of combination thyroxine and triiodothyronine (T4 and T3) treatment. Further research, including randomized clinical trials, is required to resolve these issues.

\section{Q. What are the limitations of current newborn screening programs for congenital hypothyroidism?}

Testing for congenital hypothyroidism was added to existing newborn screening programs in the mid1970s. These programs have been incredibly successful, allowing for thyroid hormone treatment starting at a few weeks of life. 'There are three screening test approaches to detect congenital hypothyroidism:

- Primary T4-reflex thyroid-stimulating hormone;

- Primary TSH; and

- Combination T4 and TSH.

Each of these test approaches detects congenital primary hypothyroidism at an approximate rate of $1: 2,000$ to $1: 4,000 .^{2}$

Congenital hypothyroidism typically presents with low T4 and elevated TSH levels. By contrast, some cases do not have elevated TSH levels on initial testing during 1-3 days of life, whereas on repeat testing after a few weeks of life, TSH is elevated—-the so-called "delayed TSH elevation." ${ }^{\prime 3}$ This pattern in thyroid function tests is most commonly present in preterm, low-birthweight babies, or acutely ill babies admitted to an intensive care unit. Infants with a delayed TSH elevation will be missed by primary TSH test programs that collect only a single specimen. Several newborn screening (NBS) programs collect two routine specimens in all newborns, while others added a "discretionary" second specimen in selected populations of newborns, such as babies born preterm. Experience has shown that most of the cases of congenital hypothyroidism characterized by delayed TSH elevation, are 
transient. However, the general practice is to treat such cases until the hypothyroidism resolves, to protect the developing brain. To detect cases of delayed TSH elevation, NBS programs would need to collect a routine second specimen, particularly in the groups at risk.

Some consideration has been given to the detection of congenital secondary (central) hypothyroidism. Although less common than congenital primary hypothyroidism (incidence 1:25,000), studies show that babies with congenital central hypothyroidism have approximately the same degree of hypothyroxinemia as primary hypothyroidism. ${ }^{4}$ Early detection and treatment likely would have the same benefit as with primary hypothyroidism, with the added benefit of early testing for other pituitary hormone deficiencies. Only the primary T4-reflex TSH or combined T4-TSH test approach have the potential to detect congenital central hypothyroidism. Thus, to detect this condition, primary TSH test programs would have to switch to one of the other test approaches.

In addition to early detection and treatment, ongoing monitoring of serum thyroid function is important, allowing L-T4 dose adjustments as the baby grows. The brain has a critical dependence on thyroid hormone during the first 2-3 years of life. Studies show that a significant proportion of infants with congenital hypothyroidism are lost to follow-up and do not refill their L-T4 prescriptions as expected. ${ }^{5}$ Few NBS programs have the resources to assure timely monitoring of thyroid function together with clinical evaluation, which are both necessary to assure optimal L-T4 dose adjustments. NBS programs in concert with primary care physicians need to develop patient registries, with built-in reminders when families miss lab test monitoring, physician visits, or prescription refills.

\section{Q. What new techniques can improve diagnostic accuracy?}

The differences in incidence rates of primary congenital hypothyroidism noted above may reflect slight differences in test cutoffs. The T4 cutoff leading to a reflex TSH test is typically <10th percentile, but in some programs it is as high as $<20$ th percentile. As the serum TSH level surges after delivery (as high as 60-80 mU/L), and then falls over the first 5 days of life to $<7 \mathrm{mU} / \mathrm{L}$, all NBS programs have developed "age-related" TSH cutoffs, based on the age the NBS specimen is obtained. These cutoffs tend to vary from program to program. ${ }^{6}$ Experience has shown that programs with lower TSH cutoffs tend to report a higher incidence of congenital hypothyroidism, likely the result of detection of milder cases. Efforts toward harmonization of T4 and TSH age-related cutoffs would likely result in similar incidence and more uniform detection of cases by all NBS programs.

\section{Q. What have we learned about the milder forms of congenital hypothyroidism that are increasingly being diagnosed?}

NBS programs are increasingly detecting neonates with mild hypothyroidism or isolated hyperthyrotropinemia, defined by a mild elevation in serum TSH (typically 6-20 mU/L) and a serum-free T4 usually in the normal range for age. The most common explanation (albeit not the only one) for the increasing detection of these mild cases is lowering of the screening TSH cutoff. For example, in Ontario, Canada, the NBS TSH cutoff changed in 2006 from TSH $\geq 20 \mathrm{mU} / \mathrm{L}$ to $>17 \mathrm{mU} / \mathrm{L}$ (whole blood). A rise in the incidence of mild neonatal hyperthyrotropinemia was subsequently observed, from $10 \%$ of all cases of congenital hypothyroidism in 2000 to
$42 \%$ of all cases of congenital hypothyroidism in $2010 .^{7}$ In general, over half of infants with mild congenital hypothyroidism have been shown to have "thyroid gland in situ" by imaging. The finding of a thyroid gland in situ suggests some form of thyroid dyshormonogenesis may be the most common explanation for these mild cases. A NBS program in Northern Italy reported that 25 of 84 infants (29.7\%) with congenital hypothyroidism and thyroid gland in situ had iodide organification defects based on positive perchlorate discharge tests. ${ }^{8}$ Of those with partial organification defects, 30\% had mutations of DUOX2. As described above, prematurity is a risk factor for development of an atypical form of mild congenital hypothyroidism, which is characterized by delayed TSH elevation. The decision to initiate thyroid hormone replacement in cases of mild congenital hypothyroidism remains controversial. Few studies have investigated the effects of untreated persistent hyperthyrotropinemia in infants on neurocognitive outcomes. Although a high proportion of cases have transient hypothyroidism, most clinicians opt to treat such cases until the hypothyroidism resolves to protect the developing brain.

\section{Q. What is the optimal means of management of mild subclinical hypothyroidism in children?}

Mild subclinical hypothyroidism is defined by a slight serum TSH elevation, usually in the 5-10 mU/L range, and a normal free T4 level. Based on the National Health and Nutrition Examination Survey, subclinical hypothyroidism occurs in approximately $2 \%$ of adolescents ${ }^{9}$ thus making it the most common disturbance of thyroid function. While mild TSH elevations in the 5-10 $\mathrm{mU} / \mathrm{L}$ range may be associated with a true thyroid disorder, and in some cases precede the onset of overt hypothyroidism, many cases represent TSH elevations that are above the upper limit of normal for the assay (>+2 standard deviation) but normal for the patient. Other cases appear to be assay-dependent; repeat measurement using a different TSH assay platform may be normal. In addition, there are assay artifacts that cause falsely elevated TSH levels, including the presence of interfering heterophile antibodies (human antimouse monoclonal antibody) or macro-TSH, which is caused by a complex of TSH and an immunoglobulin. Uncommon causes of mild TSH elevation include genetic defects resulting in an abnormal TSH beta gene, resulting in reduced TSH bioactivity, or a TSH receptor gene mutation, which causes TSH resistance.

Many children with obesity undergo testing of thyroid function and are found to have mildly elevated serum TSH levels, usually in the 5-8 $\mathrm{mU} / \mathrm{L}$ range. This mild TSH elevation may represent an adaptive response to obesity, perhaps driven by elevated leptin levels, rather than true thyroid dysfunction. If these patients are able to lose weight, often the serum TSH normalizes without thyroid hormone treatment. ${ }^{10}$

In children with a low degree of clinical suspicion of hypothyroidism, one approach is to repeat the TSH measurement in another lab using a different assay method. If serum TSH is still elevated, it may be helpful to ask the lab to check for heterophile antibodies or macro-TSH. If these steps do not resolve the problem, observation with repeat serum TSH in a few months will help determine a trend. Studies show that TSH will normalize in approximately $35 \%$ of cases, mild TSH elevation will persist in $45 \%$, TSH will increase in 10\% (but with free T4 still normal), while 10\% will progress to overt hypothyroidism. ${ }^{11}$ The most common cause of progression is autoimmune thyroid disease (Hashimoto's thyroiditis). Thus, in cases of persistent TSH elevation, it is helpful to measure anti-thyroid peroxidase and anti-thyroglobulin antibodies. 
In cases of persistent subclinical hypothyroidism, it is our practice to treat children if the TSH increases to $>10 \mathrm{mU} / \mathrm{L}$, if a goiter or serologic evidence of autoimmune thyroid disease is present, or if there are convincing clinical features of hypothyroidism, such as a slowing of linear growth. ${ }^{12}$

\section{Q. What are the major challenges of levothyroxine therapy in children?}

For most children, levothyroxine treatment of hypothyroidism is relatively straightforward. Recommended initial L-T4 starting doses on a weight basis are well established and monitoring of serum TSH and free T4 will then allow adjustment of dosing to achieve euthyroidism. When serum TSH remains elevated despite recommended dosing, the most common explanation is a problem with adherence to daily administration of L-T4. This is particularly a concern with adolescents who are made responsible for taking their daily pill. When serum TSH remains elevated despite assurances of daily compliance, problems with levothyroxine absorption or accelerated metabolic clearance may explain persistent elevations. common foods in the diet or medications that are known to interfere with absorption of levothyroxine include soy protein, concentrated calcium or iron, proton pump inhibitors, and bile acid sequestrants. Common drugs that are known to increase clearance include anticonvulsants, such as phenobarbital and phenytoin and rifampin.13 In general, levothyroxine should be taken without food.

Some parents or patients will describe persistent concerns, such as fatigue, weight gain, or behavioral or learning issues despite normalization of serum TSH and free T4. Some parents will ask for "natural" thyroid hormone rather than levothyroxine, by which they mean animal-derived desiccated thyroid preparations that contain a combination of T4 and T3. Serum T3 levels are normalized by deiodinase activity in patients treated with levothyroxine. However, there is evidence that some patients with specific polymorphisms in genes that modulate the effects of exogenous T4, e.g. the C-allele of single nucleotide polymorphism rs22355544 in type 1 deiodinase (DI01), may not achieve serum T3 levels that are normal for them, perhaps explaining some persistent clinical features. ${ }^{14}$ Some studies in infants with congenital hypothyroidism treated with appropriate L-T4 doses, but with persistent TSH elevation, show that adding a small dose of T3 will normalize serum TSH. ${ }^{15}$ Recommendations regarding consideration of combination T4 and T3 use in children await further study, such as randomized clinical trials. $\square$
1. LaFranchi SH. Approach to the diagnosis and treatment of neonatal hypothyroidism. J Clin Endocrinol Metab. 2011:96:2959-67.

2. LaFranchi SH. Newborn screening strategies for congenital hypothyroidism: An update. J Inherit Metab Dis. 2010;33:225-33.

3. Woo HC, Lizarda A, Tucker R, et al. Congenital hypothyroidism with a delayed thyroid stimulating hormone elevation in very premature infants: incidence and growth and developmental outcomes. J Pediatr. 2011;158:538-42.

4. Zwaveling-Soonawala N, van Trotsenburg AS, Verkerk PH. The severity of congenital hypothyroidism of central origin should not be underestimated. I Clin Endocrinol Metab. 2015:100:E297-300

5. Kemper AR, Ouyang L, Grosse SD. Discontinuation of thyroid hormone treatment among children in the United States with congenital hypothyroidism: findings from health insurance claim data. BMC Pediatrics. 2010;10:9.
6. Kilberg MJ, Rasooly IR, LaFranchi SH, et al. Variations in approach to newborn screening for congenital hypothyroidism in the U.S. J Pediatr. 2018:192:204-8.

7. Oren A Wang MK, Brnjac L, et al. Mild neonatal hyperthyrotrophinaemia: 10-year experience suggests the condition is increasingly common but often transient. Clin Endocrinol. 2013;79:832-7.

8. Rabbiosi S, Vigone MC, Cortinovis F, et al. Congenital hypothyroidism with eutopic thyroid gland: analysis of clinical and biochemical features at diagnosis and after re-evaluation. J Clin Endocrinol Metab. 2013;98:1395-402.

9. Hollowell JG, Staehling NW, Flanders WD, et al. Serum TSH, $\mathrm{T}(4)$, and thyroid antibodies in the United States population (1988 to 1994): National Health and Nutrition Examination Survey (NHANES III). J Clin Endocrinol Metab. 2002;87:489-99.

10. Longhi S, Radetti G. Thyroid function and obesity. J Clin Res Pediat Endocrinol. 2013;5(Suppl. 1):40-4.
11. Lazar L, Frumkin RB, Battat E, et al. Natural history of thyroid function tests over 5 years in a large pediatric cohort. J Clin Endocrinol Metab. 2009:94:1678-82.

12. Bona G, Prodam F, Monzani A. Subclinical hypothyroidism in children: natural history and when to treat. J Clin Res Pediatr Endocrinol. 2013:5(Suppl. 1):23-8.

13. Garber JR, Cobin R, Gharib H, et al. Clinical practice guidelines for hypothyroidism in adults: cosponsored by the American Association of Clinical Endocrinologists and the American Thyroid Association. Thyroid. 2012;22:1-36.

14. Wiersinga WM, Duntas L, Fadeyev V, et al. 2012 ETA guidelines: the use of L-T4 + L-T3 in the treatment of hypothyroidism. Eur Thyroid I. 2012:1:55-71.

15. Paone $L$, Fleisch $A F$, Feldman $H A$, et al. Liothyronine improves biochemical control of congenital hypothyroidism in patients with central resistance to thyroid hormone. J Pediatr. 2016;175:167-172 